\title{
THE CONSERVATIVE TREATMENT OF HYDRONEPHROSIS
}

\author{
By Professor V. Dix, F.R.C.S. \\ Surgeon to the London Hospital and Professor of Surgery in the University of London
}

It is my intention in this paper to discuss the aims of the conservative treatment of hydronephrosis; to indicate the results that may be expected after conservative procedures; and to describe some operations of which I have personal experience and the types of hydronephrosis in which they can be used with reasonable expectation of permanent cure.

If it is accepted that a man with one kidney has a normal expectation of life-and there is no evidence to the contrary-it follows that nephrectomy for unilateral hydronephrosis will have uniformly good results. The diseased organ has been removed and can no longer produce symptoms. It is against this standard of complete cure that we must measure the results of conservative procedures. The only other danger-the possibility of injury to the single remaining kidneyis remote, and cannot reasonably be used as an argument for preferring conservative operations to nephrectomy.

Before considering the types of hydronephrosis and conservative operations, it is useful to have a clear conception of the kind of result that may be expected. Although a rigid classification is impossible, since one class merges imperceptibly into the next, the following list covers most of the results seen in following up a series of cases :-

I. The patient is free from symptoms, the function of the kidney normal and the shape of the pelvis within normal limits. This may be considered a perfect result. It is relatively uncommon.

2. The patient is free from symptoms and the shape of the pelvis within normal limits, but the function is still subnormal. This is a common result.

3. The patient is free from symptoms but the renal function is subnormal and the shape of the pelvis abnormal.

4. In addition to subnormal function and pelvic abnormality there is infection or some other complication or there is a return of pain.

It must also be remembered that certain conservative operations are not without risk of immediate failure. It is certainly true that in any operation in which redundant pelvis is excised, with or without detachment and reimplantation of the ureter, a persistent urinary fistula may occur, and the nephrectomy that was to have been avoided will have to be performed before the patient leaves hospital.

On theoretical grounds conservative treatment would seem to be the method of choice, if there is a reasonable chance of obtaining a result of the first type, or in less favourable circumstances one not worse than the second type. In results of the third type there was probably an error of judgment in selecting the case ; in the fourth type there can be little doubt that a primary nephrectomy should have been performed.

How is it then possible to select the right case for conservative treatment ? During the pre-operative investigations, more attention should be paid to the function of the affected kidney than to its shape in the pyelogram. This may be tested in any of the usual ways-urine urea concentration, phenol-sulpho-phthalein, or indigo-carmine-my own preference being for indigo-carmine. Too little attention is usually given to the normal kidney, which often indicates clearly the degree of permanent loss of function on the affected side. A conspicuous hypertrophy of the normal kidney, with a rapid and satisfactory concentration of indigo-carmine, always indicates a great loss of function on the affected side, and a condition that is in many cases irreversible. If the hypertrophied sound kidney has taken over most of the total renal function, the possibility that the hydronephrotic kidney will regain its original share of this function is small.

The final decision:must be postponed until the kidney has been exposed and mobilized, and it is at this stage that it is most important to remember the aims of conservative surgery, as set out above. The anatomical conditions may favour one of the conservative procedures to be described later, although the kidney tissue itself may have reached the point at which recovery will be minimal. If the bulk and consistency of the renal parenchyma confirm the indications of the preliminary investigations, that little return of function is to be 
expected, nephrectomy should be performed, even when the type of hydronephrosis is otherwise most suitable for conservative treatment. The temptation to perform a technically pleasing conservative operation is especially strong at this stage, and it is for this reason that I have stressed the importance of a clear conception of the aims of conservative surgery, and of reaching a tentative decision from the facts available before the operation.

The selection of the right case for conservative treatment depends on the experience of the surgeon and his critical judgment in assessing the results of previous cases. It is impossible to give figures for any specific test of renal function that will indicate the limits of what is practicable. I would suggest, however, as a suitable case for conservative treatment, one in which there is a fair concentration in the 15 -minute film of an excretion pyelogram in which the calyces aro recognizable as calyces and not entirely converted into rounded or oval cavities, and in which indigocarmine appears in the excretion within ro minutes of an intravenous injection, even though its concentration is never very great. The beginner in this field of surgery would do well to begin by selecting only those cases with a well-marked hydronephrosis and a minimal impairment of renal function.

In certain cases of bilateral hydronephrosis it may be necessary, as a life-saving measure, to run the risk of performing conservative operations when the renal impairment already present does not give much hope of a return to normal. Unexpectedly good results may be obtained in some of these cases.

Infection and the presence of a few small calculi-usually in the lower calyces-are not in themselves contraindications to conservative operations. Infections which would not clear up in the obstructed kidney may die out or yield to treatment when the obstruction is removed, and the calculi, in the absence of infection, will probably not recur when the pelvis is emptying normally.

\section{Types of Hydronephrosis}

The variation in the size of the pelvis in its diastolic phase is very great, and is not necessarily the same on the two sides. It is not my intention to discuss in this paper what degree of dilatation may, with justification, be called a hydronephrosis requiring treatment. It is enough to say that in all the cases on which my conclusions are based, there was at least some distortion of the shape of the calyces and. some impairment of renal function. In border-line cases I have always preferred to wait for some months, especially if the renal pain was atypical, in order to see whether the condition was progressive. It is most unlikely that the renal impairment will become so much worse in six months that a conservative operation is no longer possible, and in some cases no operation will ever be necessary. I have recently seen a patient whom I advised to have an operation in 1940 for an early hydronephrosis, although the pain was not quite typical. The air raids of that year made her decide to postpone the operation. She has had no pain during the last nine years, although the pelvic dilatation remains unchanged and the $194^{\circ}$ and I949 pyelograms are indistinguishable. It now seems probable that she will never have to have an operation, and there can be little doubt that the dilatation is only an unusually large diastolic phase in a normal pelvis.

Nor is it my intention to discuss the etiology of hydronephrosis or those cases in which there is also a hydro-ureter. It may be mentioned, however, that in some cases of hydronephrosis and hydro-ureter, division of the ureter at its lower end and re-implantation into the bladder will give good results.

In cases in which there is only a very small intrarenal pelvis and in which the calyces show most of the dilatation, a conservative procedure is not usually satisfactory. Most of the conservative procedures to be described can be applied only to cases in which there is a reasonable degree of pelvic dilatation. In a short paper it is impossible to describe all the conditions that may be found when the hydronephrotic kidney has been exposed, and I shall describe only three types of lesion in which I have found a conservative operation to be of some value :-

I. The kidney shows a pelvic hydronephrosis of moderate size, and there is some dilatation of the calyces to be seen in the pyelogram. When the kidney has been exposed and dissected, there is no apparent cause for the hydronephrosis, and no lower pole vessels are present. There are no obstructive fibrous strands surrounding the uretero-pelvic junction, and the uretero-pelvic junction itself appears to be normal, with no fibrosis in its musculature.

2. Round the uretero-pelvic junction there are many small fibrous strands that appear to be inflammatory in origin. These strands kink and distort the junction and have to be dissected away with great care before the ureter can be straightened out. In addition to the peri-ureteric fibrosis there is often well-marked hardness and narrowing of the upper $0.5 \mathrm{~cm}$. of the ureter, extending to the uretero-pelvic junction. The impression given is that the tube is a fibrous one and no longer entirely muscular.

3. There is a band of vessels coming to the 
lower pole of the kidney either causing the obstruction or increasing the obstruction already started by some other factor. After division of these vessels, if this is done, it may be found that the end of the ureter and the uretero-pelvic junction are normal and the contents of the pelvis can be pressed down into the ureter without the slightest difficulty. In other cases, however, there are many fibrous strands, as in the previous type, and these have to be dissected away before the upper end of the ureter is free from obstruction. In certain cases, that are much less common, there is also an obvious macroscopic fibrosis of the upper part of the ureter and its junction with the pelvis.

It is true that there are other possible combinations of the above types, and every case in which the surgeon is going to perform a conservative operation must be considered as a separate problem. But I have selected the above types as those which can be correlated with the operations I have found to be of value. Before describing the type of operation to be performed, it will be appropriate to mention again why errors of judgment are liable to occur in performing an excision of the pelvis and re-implantation of the ureter. This operation is so pleasing technically, and in suitable cases gives such good results, that there must inevitably be a strong bias in its favour at the time of the operation. But it is well to remember that it is technically easy to perform in those very cases in which a conservative operation is probably of very little value. It is not difficult to excise the pelvis and re-implant the ureter when the pelvis is very large and the function of the kidney considerably diminished. The result may be apparently. satisfactory when an instrumental pyelogram is performed after the operation, but it is really useless to perform an operation of this magnitude unless there is afterwards a wellmarked restoration of function, as well as a satisfactory lroking pelvis.

\section{Operations}

The three operations I have performed are as follows :-

I. Ligature and division of lower pole vessels.

2. Dilatation of the uretero-pelvic junction and temporary nephrostomy.

3. Excision of the pelvis and re-implantation of the ureter.

For all these operations the kidney may be exposed by any of the usual incisions. It will be found that a larger incision is necessary for an excision of the pelvis than for the other two operations.

I. Ligature and division of lower pole vessels.

After the kıdney has been dissected and mobilized, it may be considered that the lower pole vessels are the primary cause of the obstruction, or that they may have become an important factor in maintaining the hydronephrosis already initiated by some other mechanism. In either case, unless the vessels are very large, they should be divided between ligatures. If there is no peri-pelvic and peri-ureteric fibrosis, it should then be possible to squeeze urine from the unopened pelvis into the ureter. When this can be done easily, demonstrating the absence of permanent narrowing of the junction, nothing more need be done. When, however, the uretero-pelvic junction is surrounded by fibrous strands, a careful dissection must be undertaken, freeing both ureter and pelvis and straightening out kinks caused by the fibrosis. It will then usually be found, as in those cases without surrounding fibrosis, that the pelvis communicates freely with the upper ureter through a ureteropelvic junction of normal diameter. It is uncommon to find intra-muscular fibrosis in these cases, and I have only once had to combine dilatation with division of lower pole vessels.

After division of the vessels there is always an area of infarction at the lower pole of the kidney and its extent can be seen by an immediate change of colour in the affected area. In most cases the estimated amount of kidney affected is not more than one-sixth of the whole, and I have never seen any serious complications following this local infarction of the kidney. If the vessels are very large, and therefore likely to supply a correspondingly large amount of the kidney, It may be possible to perform an excision of the pelvis with re-implantation of the ureter on the other side of the obstructing vessels.

The proved association of hypertension with certain cases of unilateral renal disease has created doubts about the wisdom of deliberate damage to the kidney by the ligation of lower pole vessels. There is, however, no certain evidence that, in man, renal infarction causes the type of ischaemia which initiates the series of changes resulting in hypertension. For the present we have no reason to suppose that this simple operation is more dangerous than it was before the consequences of renal ischaemia were fully appreciated. The chief merit of the operation is that the pelvis and ureter are not opened. Good results may be expected, unless the compression atrophy of the kidney has advanced too far ; when it has, nephrectomy and not ligature of the vessels is the right operation.

2. Dilatation of the uretero-pelvic junction and temporary nephrostomy.

This operation can be used for those cases of fibrosis of the upper ureter and uretero-pelvic junction, in which it is clear that there is a stricture at the pelvic outlet. A complete dissection of any external fibrous strands is necessary, whether they 
are causing kinking of the ureter or not. The pelvis is then opened (preferably on the posterior surface, but if necessary on the anterior surface) by an incision in the line of the ureter. The incision need not be more than $\mathrm{I} \mathrm{cm}$. in length. The smallest gum elastic bougie that will go through the stenosed ureteric orifice is then passed into the ureter. This is followed by a succession of bougies advancing each time by one step on the Charrière scale. In a favourable case it is possible to dilate to 12 or 13 Charrière. A temporary nephrostomy is then made in the following manner (Fig. I) :-

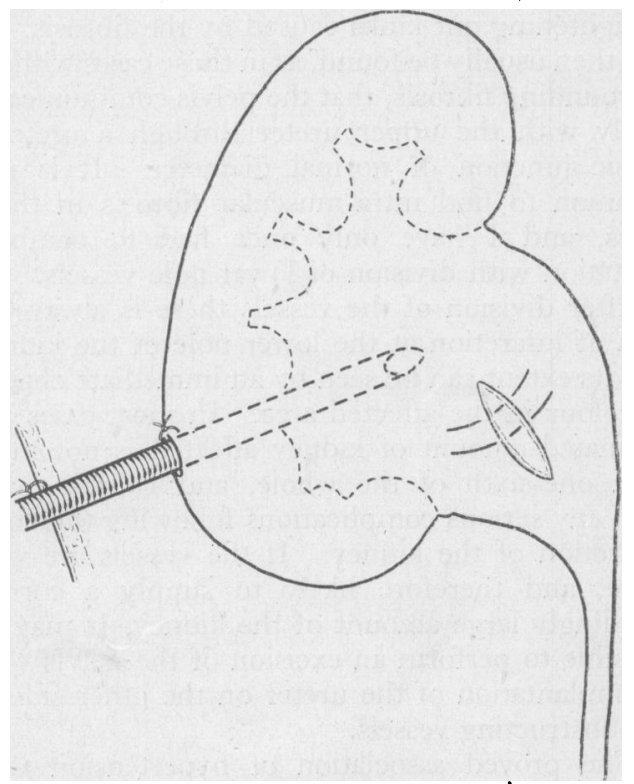

FIG. 1.-Temporary nephrostomy.

Through the opening in the pelvis a pair of forceps is introduced and the point pushed out through the renal substance so that it will emerge somewhere in the lower third of the kidney at its convex border and slightly on its posterior surface. A little haemorrhage may follow, but it is never troublesome.

The end of a whistle-tipped catheter (No. 16 Charrière, with two lateral holes) is then grasped by the forceps and drawn back into the pelvis, where it is adjusted so that it lies comfortably. When it is in the right position the catheter is held by the assistant with a pair of forceps where it emerges from the kidney. A catgut suture is passed through the catheter, which must be firmly held at this stage or it will be pulled out and a further adjustment will be necessary; the suture picks up a piece of the capsule of the kidney and the catheter is fixed. The pelvis is then sutured. Three or four cubic centimetres of fluid can be introduced through the catheter into the pelvis to see whether the pelvic suture line is water-tight or not, and to wash out any small clots.

The kidney is now returned to its position, a perinephric drainage tube inserted and the wound is sewn up. The drainage tube and the catheter draining the pelvis are allowed to come out of the wound at the most convenient place. This will often be found (in the case of an oblique incision in the loin) to be about one-quarter or one-third of the total length of the wound away from its anterior end. The catheter is then attached to the skin by a suture of whatever material is being used for the skin sutures, and it may again be washed out gently to remove small clots.

The post-operative management of the catheter and its removal will be found in the description of the next operation.

If the uretero-pelvic stricture is too narrow to admit even a small bougie, or so tough that dilatation is impossible, it may be possible to excise the constricted uretero-pelvic junction and the dilated pelvis, and to re-implant the ureter. But it is my experience, in cases of this type, that the fibrosis extends down the ureter for some distance, often so far as to prevent re-implantation without tension, when the fibrous upper part has been excised. In these circumstances a nephrectomy should be done.

During the dilatation, the uretero-pelvic junction may split. When this happens a nephrectomy will be necessary, unless the ureter can be re-implanted after the stricture and the redundant pelvis have been excised. It is uncommon for dilatation to cause splitting, and it is a risk that must be run if adequate stretching is to be.obtained. As there is rarely any other conservative procedure suitable for cases of this type, a very occasional failure must be accepted as a reasonable risk in an attempt to save the kidney.

3. Excision of the pelvis and re-implantation of the ureter (von Lichtenberg).

This operation is suitable for cases with lower pole vessels that are considered to be too large for ligature and division; for cases of stricture in which the stricture is limited to the uretero-pelvic junction and does not encroach upon the ureter; and for any cases of hydronephrosis of the pelvic type in which neither of the above simple operations is suitable. When the kidney has been exposed, it is freed from the perinephric tissue and a complete dissection of the renal vessels and the upper part of the ureter is made. If it appears that the hydronephrosis has been due to kinking of the ureter over a vessel, it may be possible to divide the ureter below this point and to re-implant it into the reconstructed pelvis on the other side of the obstructing vessel. When there is any peri- 


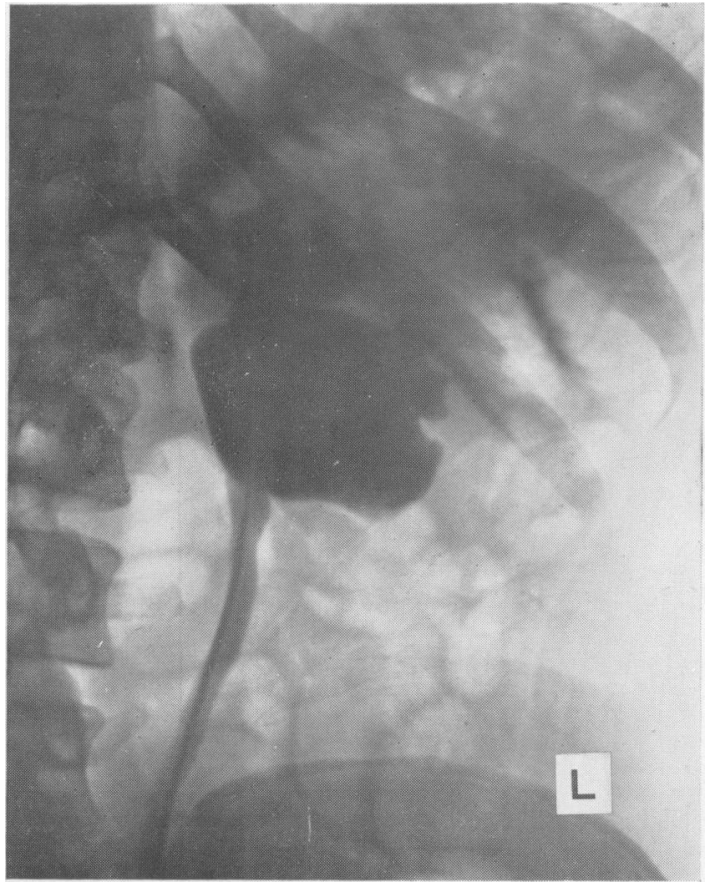

FIG. 3.-B.R. Pre-operative pyelogram (October, 1932).

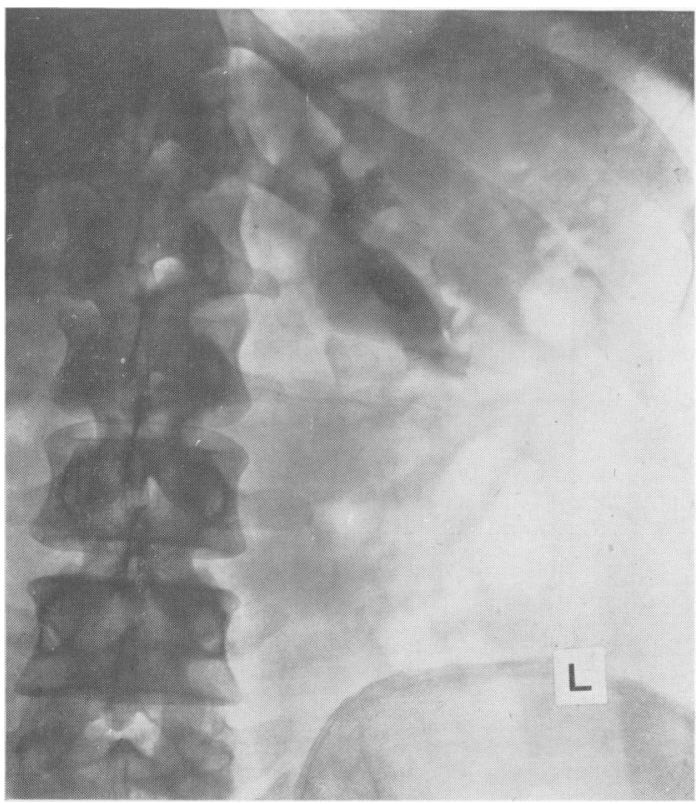

FIG. 5--B.R. Excretion pyelogram (15-minute film) I $6 \frac{1}{2}$ years after operation (May, 1949). This patient had a normal pregnancy five years ago. In May, 1949, indigo-carmine was excreted in good concentration from the kidney in five minutes.

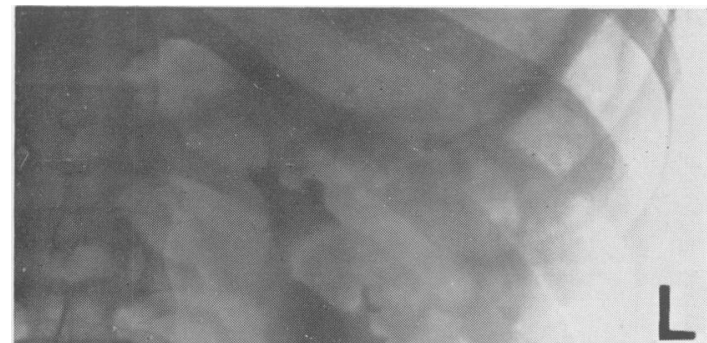

Fig. 4.-B.R. Pyelogram io months after excision of pe!vis and re-implantation of ureter (August, I 933).

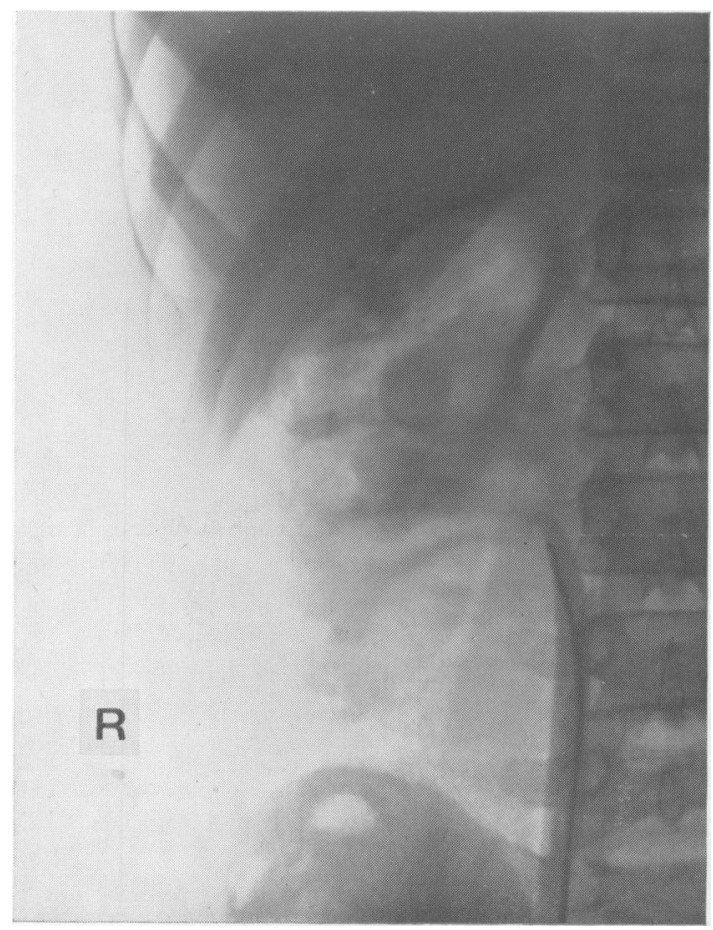

FIG. 6.-Q.S. Pre-operative pyelogram. 


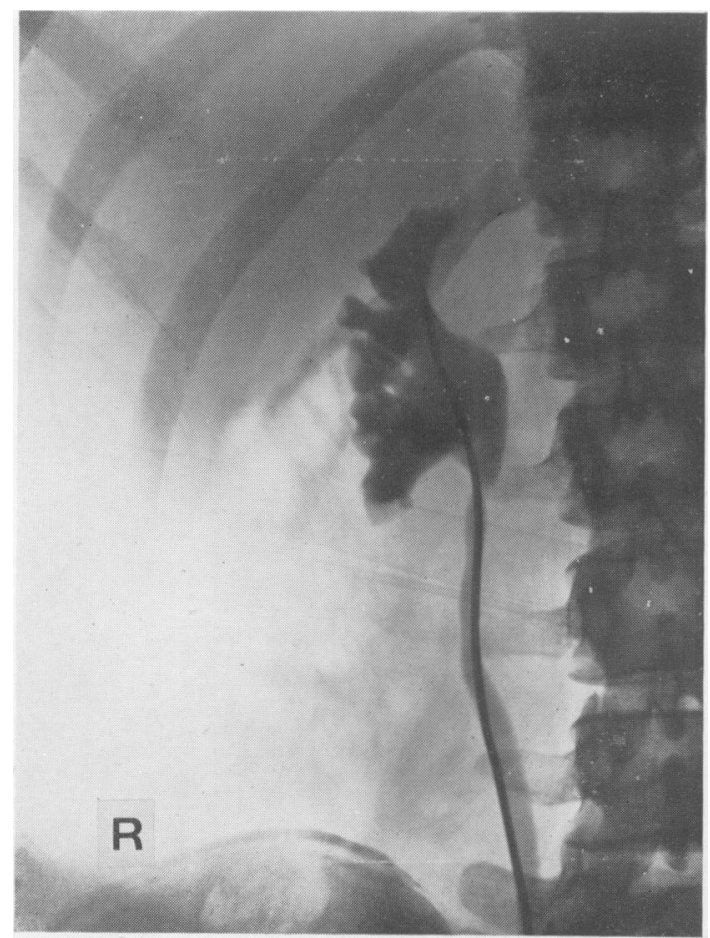

FIG. 7.-Q.S. Pyelogram six years after dilatation of uretero-pelvic junction and temporary nephrostomy. Indigo-carmine was excreted in good concentration from the kidney in four minutes.
FIG. 9.-G.K. Pyelogram I 5 months after ligature and division of lower pole vessels. Indigo-carmine was excreted in four minutes, but the concentration was not normal. Although this patient was free from symptoms, and the calyces almost normal, the pelvis is still abnormal and the renal function subnormal. Time will show whether it would have been better to perform nephrectomy.

(This figure, together with Nos. I, 3, 4 and 8, are reproduced by kind permission of the Editors of the Proceedings of the Royal Society of Medicine.)

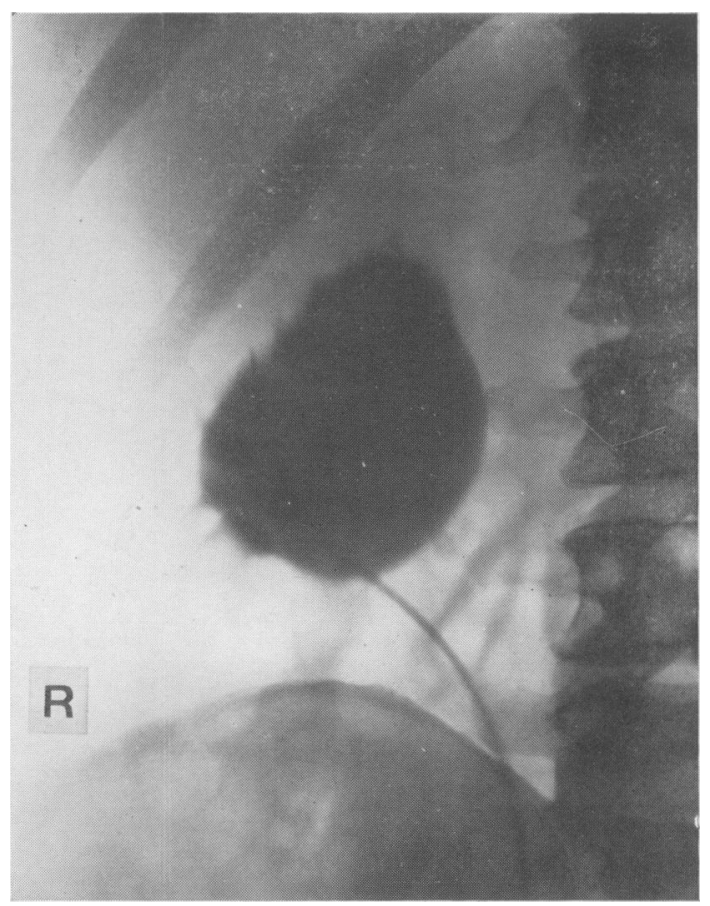

I'lg. 3.-G.K. Pre-operative pyelogram.

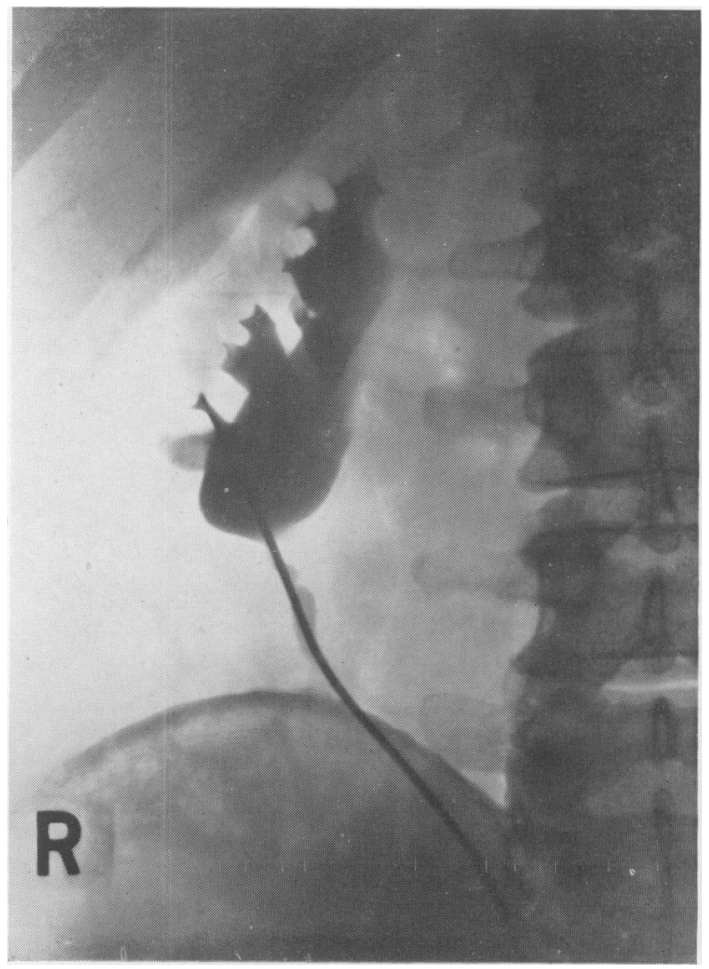


nephritis and peri-ureteritis, the fibrous strands must be carefully divided until the pelvis and the ureter are completely bare and free from adhesions.

The operation itself is performed as follows (Fig. 2) :-

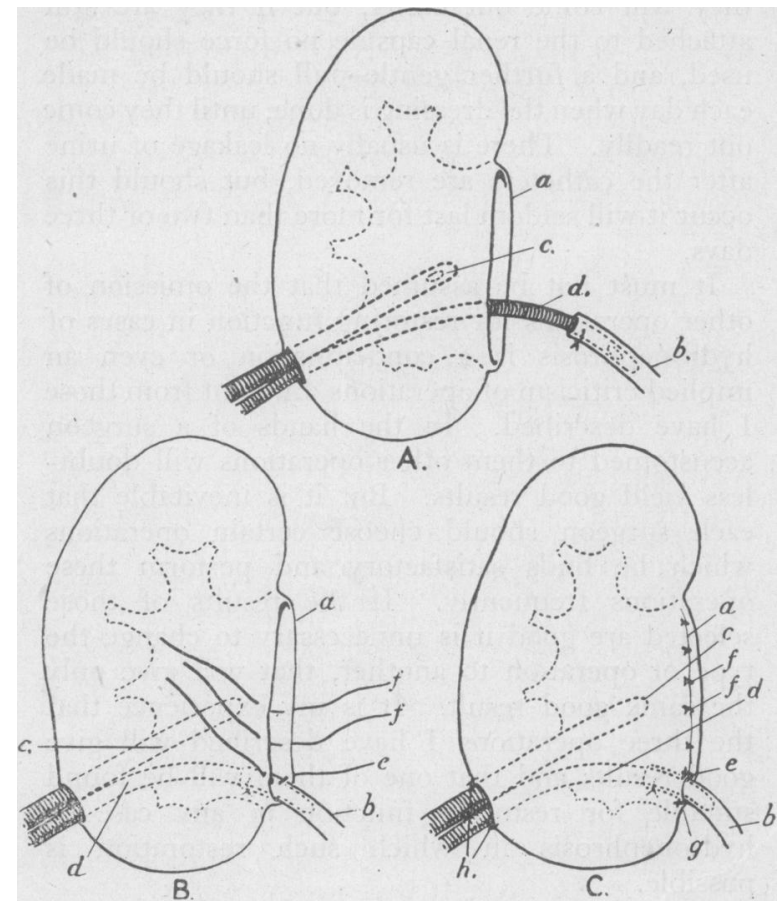

FIG. 2.-Excision of pelvis and implantation of ureter. 'Maingot's Post Graduate Surgery,' p. 2843 (by permission of Medical Publications Ltd.). (A) $a-$ Cut edge of pelvis. $b$-Ureter. $c-16 \mathrm{Ch}$. catheter in pelvis. $d-12 \mathrm{Ch}$. catheter sutured into ureter. (B) $a, b, c, d$, as in (A). e-First suture attaching ureter to lower angle of pelvis. $f f$-Sutures through cut edge of pelvis (not tied). (C) $a, b, c, d$, as in (A). $e-$ First suture attaching ureter to lower angle of pelvis (tied). $f f$-Sutures through edges of pelvis (tied). g-Extra suture attaching ureter to new pelvis. $h$-Sutures attaching each catheter separately to renal capsule.

The ureter is divided at its junction with the pelvis or, if there is an obstruction at the ureteropelvic junction, a little below this point. Either before or after division of the ureter, the pelvis is opened between two traction sutures by an incision running in the line of the ureter towards the kidney. The ureter is held by one suture which was inserted before it was divided. The pelvis can at this stage be trimmed and the superflous pelvic tissue removed. Most of the anterior and posterior surfaces should be removed, leaving enough for easy suture without tension. A pair of curved forceps is now inserted through the opened pelvis into the lower main calyx and out through the kidney substance, and two whistle-tipped rubber catheters, a No. 16 and a No. 12 Charrière, are grasped by the forceps and are drawn back through the kidney substance into the pelvis. The smaller catheter is pulled further through and inserted into the upper end of the ureter for a distance of about I. $5 \mathrm{~cm}$. A suture is then passed through the catheter and the ureter, fixing the catheter in the lumen of the ureter. This suture should be somewhere in the first centimetre of the ureter. As soon as the catheter is fixed it is pulled gently back until the upper end of the ureter lies inside the opened pelvis. About $1 \mathrm{~cm}$. of the ureter should lie in the pelvis at its lowest point, usually in the neighbourhood of the old uretero-pelvic junction. A suture is then passed, picking up first one wall of the divided pelvis, next the wall of the ureter and then the other wall of the divided pelvis. When this suture is tied on the posterior surface it will be obvious that the ureter is firmly attached to what will be the lower angle of the new pelvis. At this stage the ureter and the pelvis may be rotated and another suture inserted in the same way. It is important that these sutures should pick up only the cut edges of the pelvis and the ureteric wall and not the splinting catheter. The No. 16 catheter is then aajusted until it lies comfortably in the new pelvis and the latter is reconstructed by suturing together the cut edges. The two catheters are now fixed separately to the capsule of the kidney by passing one suture through each catheter and the capsule of the kidney. Care must be taken when passing these sutures not to pull the catheters out owing to the resistance of the rubber to the passage of the needle.

If the kidney lies well when replaced in its cavity, and there is no excessive movement on respiration, nothing further need be done. If, however, the kidney seems to move too freely with respiration, or if the vessels are long, thereby allowing the kidney a large range of movement, it is better to fix it at this stage. This can be done by decapsulating the upper two-thirds of the kidney, pushing it up under the ribs and fixing it in this position, by a simple catgut suture passed through its lower pole and attached to the upper angle of the wound. A corrugated rubber drain is placed outside the kidney in contact with the suture line in the reconstructed pelvis. The abdominal wall and the skin are then sutured in the ordinary way and the two catheters fixed at their points of exit from the wound. It will be seen that the No. 16 catheter, which is the main drainage catheter and on which the success of the operation depends, is attached at two points - at its exit from the kidney, where it is attached to the capsule, and also where it emerges from the skin incisions. The catheter in the ureter is fixed at three points; there is one 
suture attaching it to the ureter itself, another attaching it to the capsule of the kidney where it leaves its posterior border and a third suture attaching it to the skin. Before the abdominal wall is sutured the reconstructed pelvis should be tested in order to see that it is water-tight. This is done by injecting not more than 3 or $4 \mathrm{cc}$. of normal saline into the pelvis through the larger catheter.

The after-treatment is the same as that of any other kidney operation, with one exception. During the first few days after the operation, and particularly during the first 24 hours, great care must be taken to see that the drainage tube does not become blocked. There is often a little bleeding into the pelvis from its cut edges or from the hyperaemic mucosa, and small clots may form which block the holes of the catheter. If this obstruction is not removed, the pressure inside the pelvis may become so great that the stitches give way with the formation of a urinary fistula. The catheter should therefore be examined every hour during the first 24 hours in order to see that it is draining freely, and every two hours for the next 24 hours. At the end of this time the urine is usually no longer even faintly blood-stained, and no complications are likely to occur. Should the tube become blocked it can easily be cleared by gentle syringing with normal saline, or suction may be applied with the syringe. Any amount up to 4 cc. may with safety be injected into the reconstructed pelvis. The corrugated rubber drain should be removed between the fifth and seventh days, and the two catheters that are in the kidney itself may be removed between the tenth and fourteenth days. On the tenth day the skin stitches should be removed and a gentle effort made to pull out the catheters. If they are loose they will come out easily, but if they are still attached to the renal capsule no force should be used, and a further gentle pull should be made each day when the dressing is done, until they come out readily. There is usually no leakage of urine after the catheters are removed, but should this occur it will seldom last for more than two or three days.

It must not be assumed that the omission of other operations for restoring function in cases of hydronephrosis is a condemnation or even an implied criticism of operations different from those I have described. In the hands of a surgeon accustomed to them other operations will doubtless yield good results. But it is inevitable that each surgeon should choose certain operations which he finds satisfactory and perform these operations frequently. If the results of those selected are good it is unnecessary to change the type of operation to another, that will give only the same good result. It is my experience that the three operations I have described will give good results and that one of them will be found suitable for restoring function in any case of hydronephrosis in which such restoration is possible. 